This document is the Accepted Manuscript version of a Published Work that appeared in final form in Organic Electronics, copyright @ Elsevier after peer review and technical editing by the publisher. To access the final edited and published work see http://dx.doi.org/10.1016/j.orgel.2017.05.038

This manuscript version is made available under the CC-BY-NC-ND 4.0 license http://creativecommons.org/licenses/by-nc-nd/4.0/

\title{
Visible Light-Emitting Host-Guest Electrochemical Cells Using Cyanine
}

\section{Dyes}

Sandra Jenatsch ${ }^{1,2}$, Lei Wang ${ }^{1,3}$, Nicolas Leclaire ${ }^{1,2}$, Erwin Hack $^{4}$, Roland Steim ${ }^{1}$, Surendra B. Anantharaman $^{1,2}$, Jakob Heier $^{1}$, Beat Ruhstaller ${ }^{5,6}$, Lieven Penninck ${ }^{6}$, Frank Nüesch ${ }^{1,2}$, Roland Hany $^{1, *}$

${ }^{1}$ Empa, Swiss Federal Institute for Materials Science and Technology, Laboratory for Functional Polymers, CH-8600 Dübendorf, Switzerland.

${ }^{2}$ Institut des Matériaux, Ecole Polytechnique Fédérale de Lausanne, EPFL, Station 12, CH1015 Lausanne, Switzerland.

${ }^{3}$ Zürich University of Applied Sciences, Institute of Chemistry and Biological Chemistry, Einsiedlerstrasse 31, CH-8820 Wädenswil, Switzerland.

${ }^{4}$ Empa, Swiss Federal Institute for Materials Science and Technology, Reliability Science and Technology, CH-8600 Dübendorf, Switzerland.

${ }^{5}$ Zürich University of Applied Sciences, Institute of Computational Physics,

Technikumstrasse 9, CH-8401 Winterthur, Switzerland.

${ }^{6}$ Fluxim AG, Technoparkstrasse 2, 8406 Winterthur, Switzerland.

*Corresponding author.

Correspondence should be addressed to Dr. Roland Hany

Empa / Laboratory for Functional Polymers

CH-8600 Dübendorf - Switzerland

E-mail roland.hany@empa.ch 
KEYWORDS light-emitting electrochemical cell, cyanine dye, host-guest, resonance energy transfer, visible emission

ABSTRACT: Light-emitting electrochemical cells (LECs) can be fabricated as a single emissive organic/salt layer sandwiched between two electrodes, offering cost-effective next generation signage and lighting applications. Cyanine dyes are especially attractive to exploit the low cost potential of LECs. Cyanines denote a large class of fluorescent organic salts with tuneable emission wavelength, inherent conductivity for ionic and electronic charges, and many cyanines are commercially available at low cost. We systematically tested a set of cyanine dyes for visible emitting LECs. To circumvent non-radiative quenching processes in pure cyanine films (monomer fluorescence quantum yields, PLQE, $<1.5 \%$ ) we exploited the efficient resonance energy transfer (RET) from cyanine host to cyanine guest molecules $($ PLQE maximum $=16.2 \%)$. The analysis indicated that specific host-guest interactions or a parallel energy transfer channel to host dimers can reduce the guest PLQE, despite a generally high RET efficiency in cyanine host-guest systems. By comparing single component with host-guest LECs, we found that the PLQE enhancement directly translated into the device efficiency increase, and red-emitting host-guest LECs with an external quantum efficiency (EQE) of $0.36 \%$ were achieved, close to the theoretical EQE maximum $(0.81 \%)$. Chemical approaches that provide sterically demanding (to increase the PLQE) and high bandgap (for emission at smaller wavelengths) cyanines at low cost promise further progress in the field.

\section{INTRODUCTION}

Organic light-emitting electrochemical cells (LECs) have been presented as a low-cost alternative to the more frequently used organic light-emitting diodes (OLEDs). Due to their 
particular operation principle, they can be run as a single, thickness-tolerant layer between two air-stable electrodes [1-4]. The fundamental requirement for their operation is the combined electronic and ionic transport within the active layer. When a bias is applied to the device, the ionic charge carriers drift to the respective electrodes where they facilitate electronic charge injection [5]. The formation of $\mathrm{p}$ - and n-doped zones at the anode and cathode, respectively, ensures Ohmic contacts and leads to regions with a high conductivity which provide a surplus number of electrons and holes to the central intrinsic region where light emission occurs [6,7].

The active layer in LECs can be composed of an emissive polymer doped with a salt and an ion transporting electrolyte. Alternatively, ionic transition metal complexes (iTMCs) that possess combined ionic and electronic charge transport ability have been used $[4,8,9]$. While the former may suffer from phase separation issues $[10,11]$, the latter are expensive due to the central transition metal unit which limits their use for large area lighting applications. Recently, neutral and ionic small molecules have been employed as active components in LECs [12-19]. They are considered as an easy-to-purify [12] and low-cost [13] alternative to the more investigated polymer and iTMC materials.

Often, the low radiative quantum efficiency of organic semiconductors in a solid film presents a drawback in their use as emissive material [13]. To reduce the light self-quenching effects in single-component devices, LECs based on a host-guest approach have been presented, a concept that is frequently employed in OLEDs. For this approach a small bandgap material (guest) is blended in low concentrations into a larger bandgap material (host). While the host ensures the electronic charge transport, the emission originates from the guest. The formation of excitons on the guest can either occur through energy transfer from the host or through sequential charge trapping [20]. Efficient energy transfer via Förster or Dexter mechanisms requires a large overlap of guest absorption and host emission or of the wave functions of host and guest electrons, respectively. For charge trapping of both holes and electrons, the HOMO 
and LUMO of the guest material need to lie within the bandgap of the host. By using a guest that preferentially traps one type of carrier, the carrier mobilities can be adjusted and balanced in the active layer [21]. Such a special host-guest LEC has been presented by Liao et al [22]. They ascribed the improvement in performance upon doping to a more centered emission zone rather than to a higher radiative quantum efficiency.

Host-guest LECs with an improved efficiency due to an increase in radiative quantum yield have been demonstrated for pure iTMC systems [20,23] and by combining both polymer and iTMC [24,25]. Cyanine dyes have also been employed in LECs using the host-guest approach $[13,26,27]$. For LEC applications, cyanines are interesting due to their ionic nature and their commercial availability at low cost. Many cyanines are commercially available in a typical price range of 50 USD per gram. Therefore, these dyes are much cheaper than the commonly used polymers or triplet emitting rare earth complexes. As a drawback, cyanines are fluorescent materials and $75 \%$ of the excitons cannot be harvested in the radiative recombination process. A cyanine dye was added as fluorescent dopant to either an iTMC [26] or a thermally-activated delayed fluorescent small molecule [27]. A near-infrared emitting host-guest system was presented in which both components were cyanine dyes [13]. The large extinction coefficients of cyanines result in very high Förster transfer integrals giving rise to efficient energy transfer for low guest concentrations.

Here we use cyanine dyes in LECs with emission maxima in the visible spectral range. A set of low-priced cyanine dyes was selected, including mono- and trimethine dyes, with which the visible emission range is well covered. Combinations of host and guest materials were investigated in different doping concentrations and analyzed by comparing with the corresponding Förster radius. The best host-guest system was then employed as active material in LECs. Devices were tested using constant current operation with different amplitudes and compared to the theoretical external quantum efficiency (EQE) limit. Using 
simulations, the outcoupling efficiency was estimated for different junction positions and widths.

\section{EXPERIMENTAL SECTION}

The structures of the investigated cyanine dyes are shown in Figure 1. Dyes 1 (Sigma Aldrich) and 3a (FEW Chemicals) were received with $\mathrm{I}^{-}$as the charge compensating counter ion, $3 \mathrm{~b}$ (Synthon Chemicals) with the anion ethyl sulfate. For these dyes the counter ion was exchanged to $\mathrm{PF}_{6}^{-}$before use (see Supporting Information, SI, for synthesis details). Dyes 3c and $3 \mathrm{~d}$ were purchased from FEW Chemicals and were used without further purification.

For sample preparation, dyes 3a-3d were dissolved in acetonitrile (ACN, Sigma-Aldrich, anhydrous, 99.8\%). Pure dye and host-guest systems involving dye 1 were prepared from N,N-dimethylformamid (DMF, EMD Millipore Seccosolv®, dried) because of the poor solubility of dye 1 in ACN. For the blend films, each dye was first dissolved individually and then mixed in the desired ratio. Dye concentrations and ratios are given in weight percent, $w t \%$. For attenuation and photoluminescence (PL) measurements of pure dyes and blend films, the filtered $1 \mathrm{wt} \%$ solution was spin coated on glass at $6000 \mathrm{rpm}$ under nitrogen atmosphere. An additional PEDOT:PSS (Heraeus Clevios ${ }^{\mathrm{TM}}$ P VP AI 4083) layer was introduced between glass and dye for samples containing dye 1 to improve the homogeneity of the film. Attenuation was measured using a Varian Cary $50 \mathrm{UV}-$ vis spectrophotometer. PL measurements were performed on a Horiba Jobin Yvon Fluorolog spectrometer including an integrating sphere for quantum efficiency measurements.

LECs were fabricated on pre-patterned ITO substrates (Geomatec, $15 \mathrm{Ohms} \mathrm{square}^{-1}$ ) which were ultrasonicated in acetone, isopropanol, detergent and deionized water before use. A 80 $\mathrm{nm}$ thick PEDOT:PSS layer was spin coated on the ITO substrates and heated to $120^{\circ} \mathrm{C}$ for 10 min inside a nitrogen filled glovebox to remove residual water. The dye layer was coated from $3 \mathrm{wt} \%$ ACN or DMF solution at $6000 \mathrm{rpm}$, leading to a film thickness of about $80 \mathrm{~nm}$. 
PEDOT:PSS and dye layer thicknesses were measured using a profilometer (Ambios XP1). An Al top electrode was vapor-deposited at a base pressure of $4 \times 10^{-6}$ mbar through a shadow mask defining cells with an area of 3.1 and $7.1 \mathrm{~mm}^{2}$, respectively. The LECs were biased with different constant currents using a Keithley 2400. The transient luminance was measured with a Konica Minolta luminance meter LS-110 equipped with a close-up lens No. 110. Electroluminescence (EL) spectra were recorded using an integrating sphere connected to an Ocean Optics QE Pro spectrometer. Optical simulations were performed using the commercially available simulation software setfos (version 4.4, www.fluxim.ch). Optical constants $n$ and $k$ of dye $3 b$ and the $3 b: 3 d(99.5: 0.5)$ blend were measured by spectroscopic ellipsometry (M-2000 VI, J.A.Woollam Co.) and fitted by Gaussian oscillators (see SI). The optical constants of all other layers for simulating the full LEC stack were taken from the setfos database.

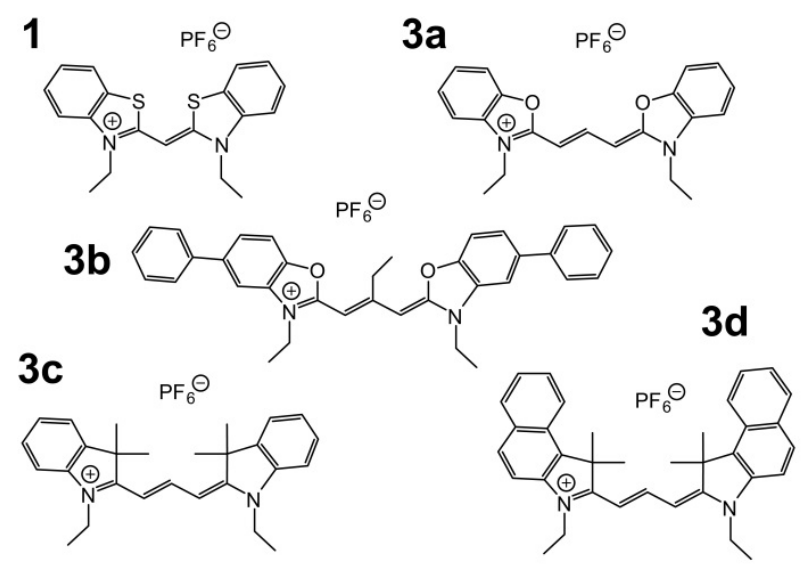

Figure 1. Molecular structures of the investigated dyes.

\section{RESULTS}

To explore the potential of cyanine dyes as active materials in visibly-emitting LECs, 5 dyes covering a large fraction of the visible spectrum were selected (Figure 1). The number of commercially available and cheap monomethine cyanine dyes is limited, therefore the lower 
wavelength limit was restricted by dye 1 . We included dye 3 a for completeness but note that coated thin films were unstable over time (see SI). Therefore, dye 3a was not considered when investigating the host-guest approach described below. The film attenuation and PL spectra are shown in Figure 2. Typically, the PL of cyanine monomers is effectively quenched by surrounding centrosymmetric non-fluorescent dimers and H-aggregates. If only the monomer contribution to the PL quantum efficiency (PLQE) is considered values in the range of $1 \%$ were measured for all investigated dyes (see Table 1), in agreement with the few available literature data [13]. In contrast to PL spectra in solution, solid state spectra of cyanine dyes can exhibit a second emission shifted by around $100 \mathrm{~nm}$ to the red. This phenomenon is observed for dyes $1,3 \mathrm{c}$ and $3 \mathrm{~d}$, although the ratio of the monomer to the second emission and its shape was rather different for the different dyes. The second emission originates from a low fraction of emissive dimers that are present in a twisted configuration [28]. If a second emission is present and large in intensity, as for the dyes $3 \mathrm{c}$ and $3 \mathrm{~d}$, the overall PLQE can be well above $1 \%$. Host-guest solid films present an opportunity to reduce non-radiative quenching and to obtain higher PLQE. This approach was used in the following to find the best system - with respect to high solid film PLQE - for LECs. 

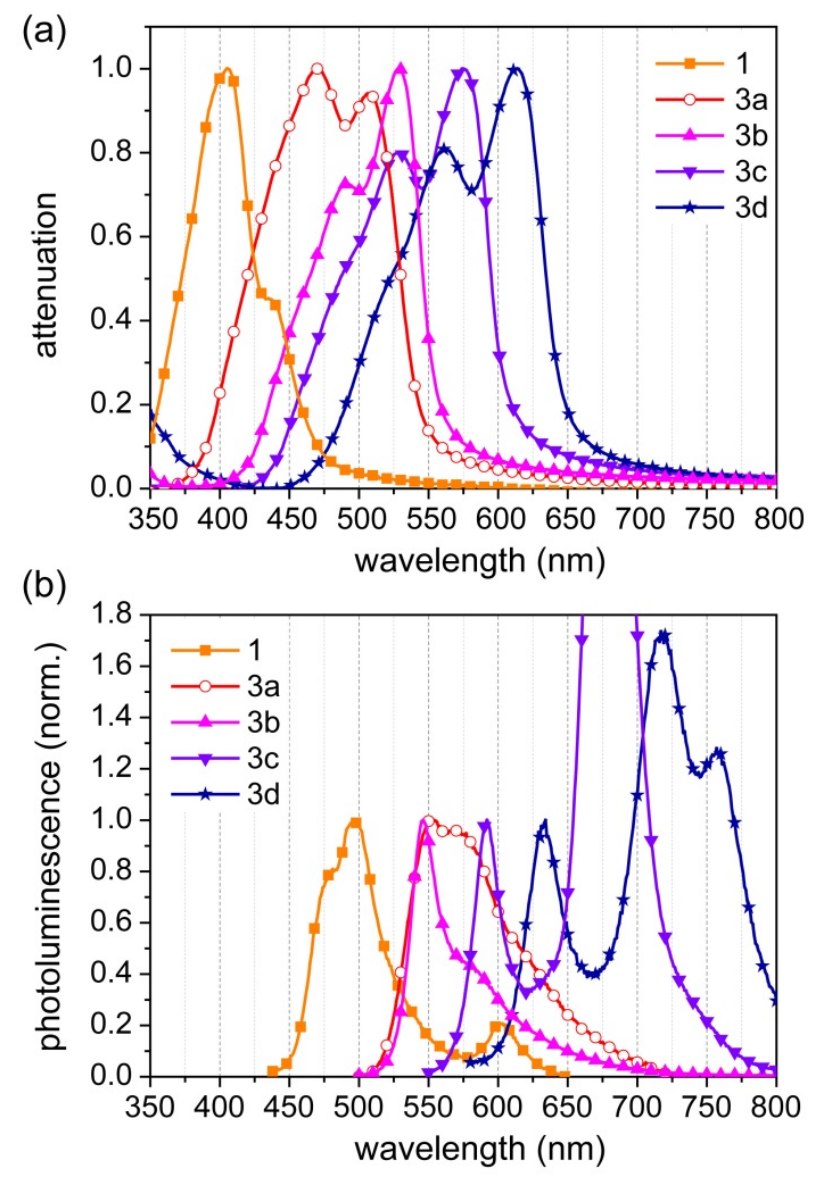

Figure 2. Normalized attenuation (a) and photoluminescence (b) spectra of pure dye films.

Energy transfer from host to guest cyanines is ascribed to Förster resonance energy transfer (FRET). In principle, Dexter transfer could also play a role. However, for fluorescent materials such as cyanine dyes, FRET has been shown to be more efficient [29]. FRET efficiency depends significantly on the large spectral overlap between host emission and guest absorption. All cyanine host-guest systems were tested that obey this requirement for FRET. For each host-guest system film PL spectra with different guest concentrations were measured and the PLQE was evaluated. As an example, PL spectra of $3 b: 3 d$ host-guest films with various guest concentrations are shown in Figure 3a. Already small 3d guest concentrations resulted in efficient energy transfer and high fluorescence intensity from the guest while the remaining host emission gradually disappeared. The guest emission increased for small additions but decreased again for concentrations higher than $0.5 \mathrm{wt} \%$. This value therefore 
presents the optimum between increased FRET, due to a closer proximity of host and guest, and low self-quenching rate because of well separated guest molecules. The inset in Figure 3a shows that a maximum PLQE of $16.2 \%$ can be obtained if $0.5 \mathrm{wt} \%$ of dye $3 \mathrm{~d}$ was mixed into the $3 \mathrm{~b}$ host matrix. This represents an increase of the PLQE of more than 10 times compared to the pure host. For a near-infrared emitting cyanine-cyanine host-guest system, a similarly large increase in PLQE (27\%) has been reported [13]. A high PLQE of 43.1\% was reported for $3 \mathrm{c}$ dispersed in a matrix of a charged carbazole derivative [27].

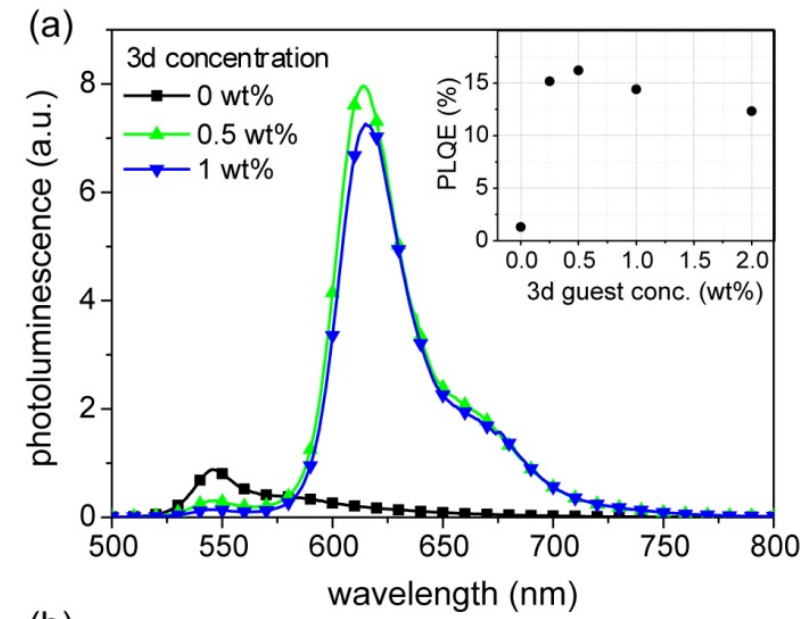

(b)

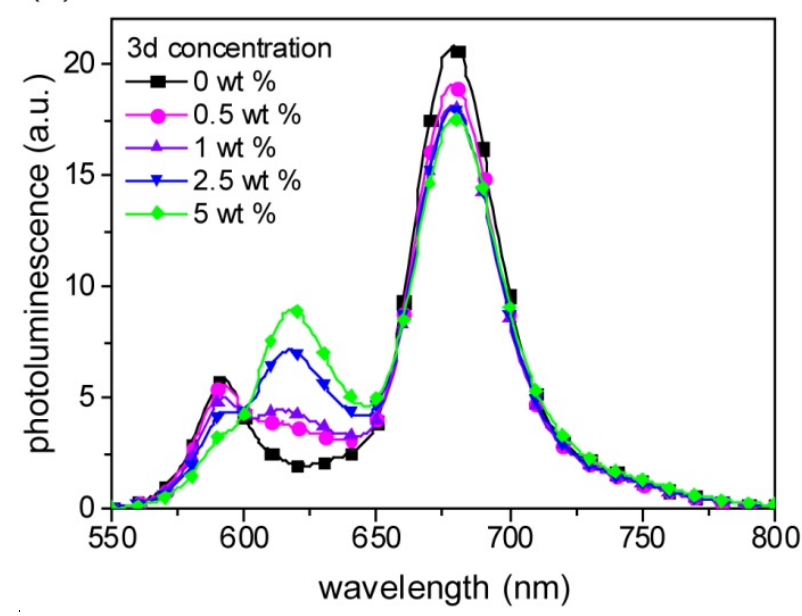

Figure 3. Photoluminescence spectra of (a) $3 b: 3 d$ and (b) $3 c: 3 d$ host-guest films with various concentrations of the guest. In (a) the inset shows the PLQE. The highest guest emission and PLQE value was observed for a guest concentration of $0.5 \mathrm{wt} \%$. 
In Figure 3a, we note the gradual shift in peak emission to higher wavelengths with increasing doping concentration. Moving further to the pure $3 \mathrm{~d}$ film results in a pronounced $20 \mathrm{~nm}$ large red shift of the $3 \mathrm{~d}$ monomer peak emission compared to the $3 \mathrm{~b}:(0.5 \mathrm{wt} \%) 3 \mathrm{~d}$ host-guest system (see Table 1). This behaviour was observed for all dye systems and can be explained by the strong aggregation tendency of cyanines with increasing concentration [30]. Table 1 lists PLQE values of pure dyes and of the optimized host-guest systems. All host-guest systems showed an improved PLQE compared to the pure hosts. However, the factor of PLQE improvement varied remarkably among different dye combinations. It has to be mentioned that the PLQE vs. guest concentration trend was fairly indistinct for host-guest systems $3 b: 3 c$ and $3 \mathrm{c}: 3 \mathrm{~d}$. Therefore, we also list the PLQE of blends with smaller guest concentrations in brackets, allowing a better comparison with the other systems as discussed below.

The Förster radius $R_{0}$ for each host-guest blend was calculated using Equation (1). This radius reflects the distance between a host and guest molecule for which the FRET efficiency is as probable (50\%) as the decay of the excited host molecule [31].

$R_{0}^{6}=\frac{9 \ln 10 \kappa^{2} \Phi_{P L}}{128 \pi^{5} n^{4} N_{A}} \int F_{D}(\lambda) \varepsilon_{A}(\lambda) \lambda^{4} d \lambda$

$\kappa^{2}$ is the orientation factor (2/3 for random orientation), $\Phi_{P L}$ is the PLQE of the host in the absence of the guest, $n$ is the refractive index (here assumed to be 2 and wavelengthindependent), $N_{A}$ is the Avogadro constant, $F_{D}$ is the area normalized emission spectrum of the host and $\varepsilon_{A}$ is the extinction coefficient of the guest. Extinction coefficients were obtained from absorption spectra in ACN solution $\left(\varepsilon_{\max }=1.6 \cdot 10^{5} \mathrm{M}^{-1} \mathrm{~cm}^{-1}\right.$ for $3 \mathrm{~b}, 1.2 \cdot 10^{5} \mathrm{M}^{-1} \mathrm{~cm}^{-1}$ for $3 \mathrm{c}$ and $3 \mathrm{~d}$ ). The extinction coefficient was measured in solution in order to mimic the diluted situation of the guest dye in a host matrix. The Förster integral and radius $R_{0}$ (from Equation 1) are presented in Table 1. Extinction coefficients were also evaluated from solid state absorption spectra. The calculated Förster radius using film extinction coefficients are very similar to the values in Table 1 (see SI). The distance between two guest molecules for 
the optimized concentrations was estimated by assuming that each guest molecule defines a sphere with a radius equal to half the average spacing between individual dopant molecules, the molecular weight of the guest and assuming a film density of $1.3 \mathrm{~g} \mathrm{~cm}^{-3}$. In Table 1 the maximum distance between a host and a guest molecule is shown, which is just half of the distance between two guest molecules, assuming their homogeneous distribution.

Table 1. Emission wavelength maxima and PLQE of pure dyes and optimized host-guest systems. The Förster radius was calculated using Equation (1) and the extinction coefficients were measured in ACN solution.

\begin{tabular}{|c|c|c|c|c|c|c|}
\hline & $\begin{array}{c}\text { Emission } \\
\text { wavelength } \\
\text { (nm) }\end{array}$ & $\begin{array}{c}\text { Guest } \\
\text { concentration } \\
(\%)\end{array}$ & $\begin{array}{l}\text { Distance between } \\
\text { host and guest } \\
\text { molecule (nm) }\end{array}$ & PLQE (\%) & $\begin{array}{c}\text { Förster integral } \\
\left(\mathrm{x} 10^{32} \mathrm{~nm}^{6} \mathrm{~mol}^{-1}\right)\end{array}$ & $\begin{array}{c}\text { Förster radius } \\
\text { (nm) }\end{array}$ \\
\hline 1 & 496,601 & & & 0.44 & & \\
\hline $3 b$ & 546 & & & 1.1 & & \\
\hline $3 c$ & 592,679 & & & $10\left(1.5^{\mathrm{a}}\right)$ & & \\
\hline $3 d$ & 634,718 & & & $4.0\left(0.8^{\mathrm{a}}\right)$ & & \\
\hline $1: 3 b$ & 535 & 0.5 & 3.3 & 7.0 & 4.1 & 2.01 \\
\hline $1: 3 c$ & 572 & 0.5 & 2.9 & 4.3 & 2.7 & 1.87 \\
\hline $1: 3 d$ & 607 & 0.5 & 3.2 & 3.3 & 2.3 & 1.82 \\
\hline $3 b: 3 c$ & 578 & $2.5\left(1^{b}\right)$ & $1.5\left(2.1^{\mathrm{b}}\right)$ & $1.9\left(1.5^{b}\right)$ & 3.9 & 2.39 \\
\hline $3 b: 3 d$ & 614 & 0.5 & 2.9 & 16.2 & 6.5 & 2.60 \\
\hline $3 c: 3 d$ & 618,680 & $5\left(1^{b}\right)$ & $1.4\left(2.5^{b}\right)$ & $11.6\left(10.5^{b}\right)$ & 1.3 & 2.79 \\
\hline
\end{tabular}

${ }^{\text {a }}$ Estimated contribution of the monomer emission to the total PLQE.

${ }^{\mathrm{b}}$ Values for non-optimized blends. 
After analyzing all possible host-guest systems, the one with the highest PLQE of $16.2 \%$, i.e. the $3 \mathrm{~b}: 3 \mathrm{~d}$ blend, was chosen for further investigations in LECs. As a comparison, devices were also fabricated with the pure host material 3b. Both LEC systems were measured by applying a constant current driving mode with different amplitudes. In Figure 4 the voltage, luminance and EQE transients are shown for two LECs operated at $10 \mathrm{~mA} \mathrm{~cm}{ }^{-2}$. For both devices the voltage was initially around $8 \mathrm{~V}$ and decreased rather fast with ongoing operation. Such behavior is commonly observed for constant current operation and is attributed to the ionic motion to the respective electrodes. This facilitates charge injection causing Ohmic contacts and doped regions which in turn lowers the voltage required for maintaining a certain current density [32]. The luminance was observed almost instantaneously for a driving current of $10 \mathrm{~mA} \mathrm{~cm}{ }^{-2}$. Continuous operation first lead to an increase in luminance until a maximum was reached after approximately 5 minutes. The transient EQE was calculated using the EL spectra of the LEC and assuming Lambertian emission. The peak values found in these specific transients were $0.015 \%$ for the pure $3 b$ and $0.29 \%$ for the $3 b: 3 d$ LEC, respectively. The improvement of a factor of $\sim 20$ is close to the measured increase in PLQE (about a factor of 15) from the host to the host-guest film. This demonstrates that the PLQE enhancement directly leads to the LEC efficiency improvement. For various LEC systems a strong EQE dependence on the applied current density has been observed which was attributed to polaronexciton and/or exciton-exciton quenching [14,33]. In order to analyze the influence of different current driving amplitudes, the current was varied over one or two orders of magnitude. In Figure 5 the extracted peak EQE values are presented as a function of applied current density. For the $3 \mathrm{~b}$ host-only device there is no clear trend with increasing current density. Due to the detection limit of the lumimeter, current densities below $1 \mathrm{~mA} \mathrm{~cm}^{-2}$ could not be measured. For the $3 b: 3 d$ device the highest performance, i.e. an EQE of $0.36 \%$, was obtained for the lowest current density. A small decrease in efficiency is observed for higher driving amplitudes. 


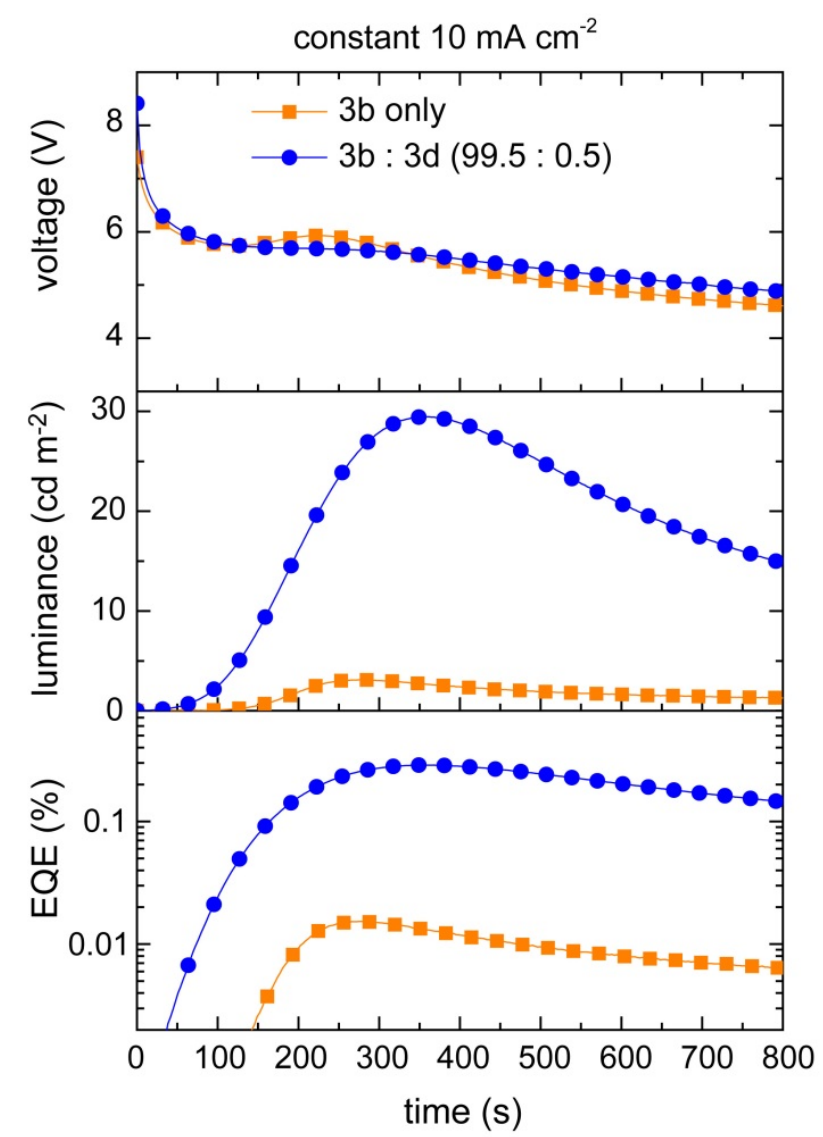

Figure 4. Transient voltage, luminance and EQE of LECs employing either pure $3 b$ host (orange squares) or the best $3 \mathrm{~b}: 3 \mathrm{~d}$ blend host-guest system (blue circles).

Even if the admixture of additional ions is not a fundamental requirement for the LEC operation based on ionic materials, several studies reported an efficiency improvement when adding extra salts $[13,34]$. In our case, the admixture of ionic liquid (IL) $\mathrm{BMIM}^{+} \mathrm{PF}_{6}^{-}$(1butyl-3-ethylimidazolium hexafluorophosphate) led to LEC devices with a faster voltage drop and luminance transient under constant current operation, but the maximum luminance never exceeded the values measured for LEC without IL (data not shown).

\section{DISCUSSION}

The presence or absence of a second emission strongly affects the total PLQE of a pure dye film. For dye $3 \mathrm{c}$ a PLQE of $10 \%$ was obtained while dye $3 \mathrm{~b}$, which only shows the emission 
of the monomer, possesses a poor PLQE of $1.1 \%$. When dye $3 \mathrm{c}$ was diluted as a guest into either dye 1 or $3 b$ the second emission was not present anymore. The absence of the large contribution of this emission also lowered the quantum efficiency of the $3 \mathrm{c}$ guest blends and explains why the total PLQE of the host-guest systems 1:3c and 3b:3c lag behind that of pure 3c. The strongly red-shifted photoluminescence originates from dimers that exist in a twisted configuration [28]. Cyanine dimers and $\mathrm{H}$-aggregates can quench the monomer emission. For a perfect parallel or antiparallel alignment of dye molecules, the low-energy excited state is optically forbidden and these aggregates are non-fluorescent. In our films, a small fraction of aggregates exists in a twisted dimer configuration which results in a non-vanishing transition dipole moment and an enhancement of the PLQE.

\subsection{PLQE and Förster transfer in host-guest systems}

A large Förster radius is desirable because already small guest concentrations enable efficient energy transfer which also minimizes aggregation-induced PL quenching among guest molecules. We first look at the cases where a small guest concentration of $0.5 \mathrm{wt} \%$ maximized the PLQE (Table 1). The highest PLQE of 16.2\% was found for the $3 \mathrm{~b}: 3 \mathrm{~d}$ blend. The merit of this system is also reflected in a large Förster radius of $2.6 \mathrm{~nm}$. The experimental host-guest distance was $2.9 \mathrm{~nm}$ which is larger than the Förster radius. This would indicate a transfer efficiency below $50 \%$ for this blend composition. The discrepancy can be explained by considering the assumption of the FRET in Equation (1). The model was developed for point-to-point transfer, it does neither account for molecules with extended dipole moments nor for diffusion of excitons in the host material. In many donor-acceptor situations the transfer efficiency is therefore more efficient than predicted from the spectral overlap of donor emission and acceptor absorption [35-37]. 
Experimentally, the energy transfer efficiency $\gamma_{E T}$ can be evaluated from the PL intensity of the pure host $I_{D}$ and the remaining PL intensity of the host in the presence of the guest $I_{D A}$ using Equation (2) [38].

$\gamma_{E T}=\frac{1}{1+\left(\frac{r}{R_{0, \text { exp }}}\right)^{6}}=1-\frac{I_{D A}}{I_{D}}$

The guest concentration for which $\gamma_{E T}$ is $50 \%$ can be used to calculate the maximal distance between a guest and a host molecule which is just the experimental Förster radius. It was found that for the $3 \mathrm{~b}: 3 \mathrm{~d}$ system the energy transfer reached $50 \%$ for a guest concentration of about $0.2 \mathrm{wt} \%$. This corresponds to an experimental Förster radius $R_{0, \exp }$ of $3.9 \mathrm{~nm}$ which is larger than the calculated value and also larger than the maximum distance between host and guest molecules in the blend that exhibits the highest PLQE. With this experimental Förster radius the efficient energy transfer from $3 b$ to $3 d$ dye in the optimized system can be rationalized.

Likewise, the host 1:guest systems can be explained by the FRET mechanism. The PLQE increase was a factor of 7.5 to $\sim 16$ when moving from the pure dye 1 to the blends. Also in these cases the calculated radii $R_{0}$ were smaller than the experimental molecular host-guest distances and we found similar experimental energy transfer efficiencies and $R_{0, \exp }$ as for the 3b:3d system. However, the absolute PLQE were considerably lower. For example, the PLQE values of the same guest $3 \mathrm{~d}$ in host $1(3.3 \%)$ and host $3 \mathrm{~b}(16.2 \%)$ differed by a factor of five. This indicates that also in the blends specific host-guest interactions occur that lead to variable non-radiative quenching of the guest.

There were two systems that required much higher guest concentrations to maximize the PLQE. The largest Förster radius was found for the 3c:3d dye blend. However, this system did not exhibit the highest PLQE and also not the highest improvement when moving from the host to the host-guest film. The required guest concentration for the highest PLQE in this blend was $5 \%$. This corresponds to a maximum distance between a host and a guest molecule 
of $1.4 \mathrm{~nm}$ which is much smaller than the calculated Förster radius. Figure $3 b$ shows the PL spectra of $3 \mathrm{c}: 3 \mathrm{~d}$ blends with different guest concentrations. The second emission of the host $3 \mathrm{c}$ is still prominently present and is redshifted with respect to the guest $3 \mathrm{~d}$ monomer emission. We speculate that an exciton created on the host can either be transferred to the $3 \mathrm{c}$ aggregate responsible for the second emission or to the guest molecule $3 \mathrm{~d}$. These two processes are competing. If a small concentration $(1 \mathrm{wt} \%)$ of the guest was added, the host monomer emission (at $592 \mathrm{~nm}$ ) reduced and the $3 \mathrm{~d}$ guest peak emission arose, while the second emission from the host remained almost unaffected. The resulting PLQE was still very close to that of the pure $3 \mathrm{c}$ film (Table 1), meaning that the desired host-guest energy transfer was not able to compete with the very effective transfer from $3 \mathrm{c}$ monomer to its dimer.

Adding more guest to the blend reduced the $3 \mathrm{c}: 3 \mathrm{~d}$ distance and increased the FRET from host to guest, resulting in a indistinct PLQE maximum for a concentration of $\sim 5 \mathrm{wt} \%$ (see Figure $3 b)$. However, the absolute PLQE increase when comparing the host $(10 \%)$ with the hostguest system (11.6\%) was moderate, also because $3 \mathrm{~d}$ aggregation with associated PL quenching can become important for higher guest concentrations. We conclude that the discrepancy between the calculated Förster radius and the much smaller distance between $3 \mathrm{c}$ host and $3 \mathrm{~d}$ guest molecules is due to a competing, very efficient energy transfer channel in the $3 \mathrm{c}$ host itself.

The other system that deviates from the FRET predictions was the $3 b: 3 c$ blend. Here, a low $(<1 \%)$ PLQE enhancement with 1-2.5 wt\% guest was found compared to the pure $3 \mathrm{~b}$ host. We observed an acceptable PL quenching of the $3 \mathrm{~b}$ host upon adding $3 \mathrm{c}$ (see SI). Therefore, we assume that also in this system the guest is populated efficiently but that the radiative quantum yield of $3 \mathrm{c}$ is strongly matrix dependent. Such matrix dependent PLQE behavior is experimentally supported by the difference between the $1: 3 \mathrm{c}(4.3 \%)$ and the $3 \mathrm{~b}: 3 \mathrm{c}(1.9 \%)$ system. Therefore, the calculated large Förster integral and radius $(2.39 \mathrm{~nm})$ for the $3 \mathrm{~b}: 3 \mathrm{c}$ system strongly overestimates the experimental achievable PLQE. 


\subsection{LEC behavior}

During operation, the voltage for our constant current driven cyanine LECs decreases steadily to below $3 \mathrm{~V}$ (see SI). Because of the relatively high driving current in Figure 4, the voltage was still at about $5-6 \mathrm{~V}$ at the point of maximum luminance and EQE. Such behavior can be due to exciton quenching when the growing doped zones approach the emission zone. Relaxation experiments were performed in order to rule out that the decrease in luminance in the $3 \mathrm{~b}: 3 \mathrm{~d}$ system is related to permanent material degradation (data not shown). Therefore, the constant current bias was switched off after the point of maximum luminance and the device was kept at open circuit for one hour. When the bias was reapplied the luminance reached the value of the first run. Similar observations were reported by others [32] and demonstrate that the luminance decay in a first place is not related to permanent material degradation.

In order to analyze the LEC performance we now turn our focus to the peak EQE values obtained for different current driving amplitudes (Figure 5). Firstly, it is observed that the factor of improvement in peak EQE ( 20) from $3 b$ host to 3b:3d host-guest LECs is the same for all current densities. In other words, the EQE trend with increasing current density is similar for both host and host-guest LECs. This confirms again that the enhancement is due to an increase of the PLQE. Furthermore, this also indicates that the ionic and electronic transports are similar in both devices, i.e. charge transport is still occurring on the host in the blend. 


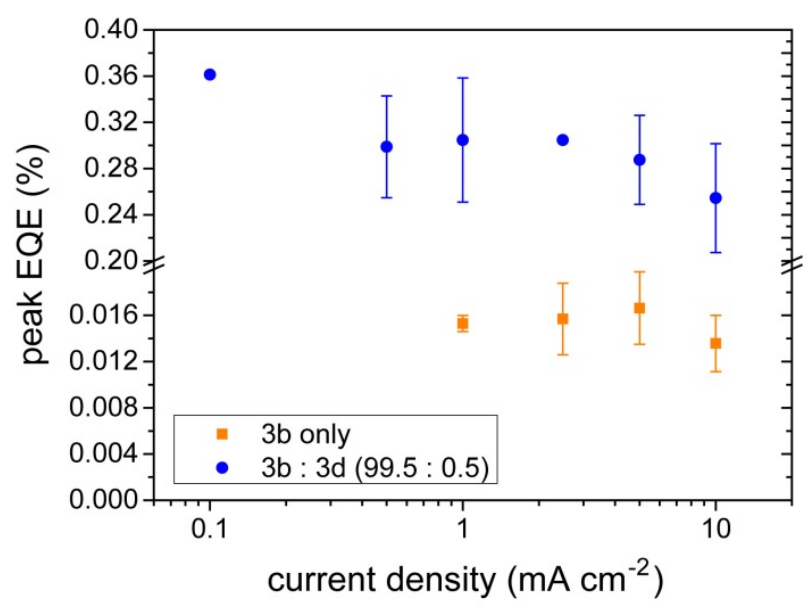

Figure 5. Peak EQE for different constant current driving for $3 b$ only (orange squares) and 3b:3d host-guest LECs (blue circles).

Secondly, there is only a slight drop in efficiency with increasing current amplitude. The number of mobile charges within the intrinsic region at $10 \mathrm{~mA} \mathrm{~cm}{ }^{-2}$ amounts to $\sim 10^{16} \mathrm{~cm}^{-3}$ assuming a charge carrier mobility of $10^{-5} \mathrm{~cm}^{2} \mathrm{~V}^{-1} \mathrm{~s}^{-1}$ [39] and an electric field across the

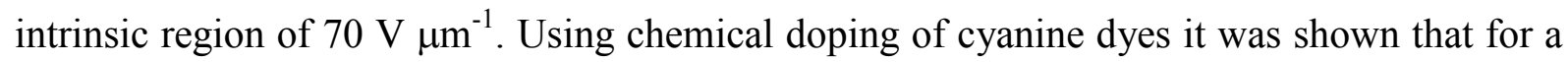
substantial PL quenching a doping concentration of some percentage is required [15]. Considering the average molecule density of $\sim 10^{20} \mathrm{~cm}^{-3}$, this suggests that the number of mobile charge carriers within the intrinsic region is not high enough for severe excitonpolaron quenching. Note that this estimation does not consider the quenching effects by the static charges in the doped regions. A pronounced EQE decrease with increasing current density was mostly found in LECs employing a triplet emitter (see SI). Additionally, polymer LECs based on the widely employed fluorescent super yellow system [40] did not exhibit a severe drop in EQE when being operated using constant current up to $10 \mathrm{~mA} \mathrm{~cm}^{-2}$ (see SI). It can be assumed that fluorescent materials are less affected by an increasing current density due to the shorter lifetimes of singlet excitons. Therefore, these devices can be operated at relatively higher current densities while still maintaining a good EQE. 
Similar as for OLEDs, the EQE is determined by four contributions [41]:

$E Q E=\gamma \eta_{s, t} q_{\text {eff }} \eta_{\text {out }}$

In Equation (3), $\gamma$ is the charge balance factor, $\eta_{s, t}$ is the spin factor, $q_{\text {eff }}$ is the effective radiative quantum efficiency and $\eta_{\text {out }}$ is the outcoupling efficiency. Due to the formation of doped regions at the electrodes the charge balance factor is considered to be unity in LECs [42]. The singlet/triplet factor is fixed by the choice of the material; for fluorescent dyes it is $25 \%$. The effective radiative quantum efficiency can be estimated from the PLQE which is $16.2 \%$ for the $3 \mathrm{~b}: 3 \mathrm{~d}$ host-guest system. However, in an operated LEC $q_{\text {eff }}$ can be lower than the value obtained from PLQE measurements. This occurs for example when the emission zone is in close proximity to the doped regions leading to quenching of excitons by stationary doped molecules.

Also, the efficiency of the light outcoupling depends delicately on the exact device architecture and the p-i-n situation within the LEC. In many cases, an outcoupling efficiency of $20 \%$ is assumed, which can be derived from ray optics for a flat emitter [43,44]. In order to analyze $\eta_{\text {out }}$ in more detail, the outcoupled light of the whole LEC stack was simulated for different emission zones within the $3 \mathrm{~b}: 3 \mathrm{~d}$ blend layer. For the simulation the dye and PEDOT:PSS layer were assumed to be transparent within the $3 \mathrm{~d}$ emission spectrum. This neglects possible reabsorption losses and sets an upper limit for the calculated $\eta_{\text {out }}$. The emission zone was assumed to be Gaussian with a certain full width half maximum $w$. The outcoupled light was calculated by sweeping the center of the emission zone through the dye layer and varying its width $w$ (see Figure 6). A maximum is observed if the emission zone is thin and close to the PEDOT:PSS interface. Increasing the width of the emission zone gradually decreases $\eta_{\text {out }}$. If the width is thicker than $\sim 30 \mathrm{~nm}, \eta_{\text {out }}$ drops below $10 \%$. Shifting the emission zone closer to the $\mathrm{Al}$ interface also leads to a decrease in outcoupling efficiency which can be reasoned by an enhanced coupling to surface plasmons of the metal electrode. Analyzing the dissipated power (see SI) indeed reveals that evanescent coupling dominates 
for thick emission zones $(>40 \mathrm{~nm})$ and positions close to the $\mathrm{Al}$ interface. For thinner emission zones $(<20 \mathrm{~nm})$ power is also dissipated via guided modes in the glass/ITO (predominantly for positions below $0.2,0=$ PEDOT:PSS/dye interface, $1=$ dye/Al interface) and in the active dye layer (especially for positions between $0.2-0.8$ ). The simulation shows that in this situation the often assumed $20 \%$ from basic ray optics is just an upper limit. $\eta_{\text {out }}$ can be considerably lower if the developing emission zone is too broad and/or not properly adjusted within the emissive layer.

A maximum EQE of $0.81 \%$ is calculated with $\gamma=1, \eta_{s, t}=0.25, q_{\text {eff }}=16.2 \%$ and $\eta_{\text {out }}=20 \%$. The best experimental EQE obtained was $0.36 \%$ and reached almost half of this theoretical limit. Even with the highest current density of $10 \mathrm{~mA} \mathrm{~cm}^{-2}$ one third of the theoretical EQE limit was achieved. Cyanine-cyanine host-guest devices with an emission in the near-infrared region reached a maximum $\mathrm{EQE}$ of $0.44 \%$ which is very similar to the results obtained within this study [13]. Higher efficiencies were achieved if cyanine dyes were mixed into a phosphorescent iTMC [26] (around 1\%) or a thermally activated delayed fluorescence (2\%) [27] host which can be rationalized by harvesting of triplet excitons and higher radiative quantum efficiencies. In general, EQE values of cyanine containing LECs are lower than those of high-efficient iTMC (8.2\%) [33] or fluorescent polymer $\left(3.2 \%, 10.6 \mathrm{~cd} \mathrm{~A}^{-1}\right.$ and 10.7 $\operatorname{lm} \mathrm{W}^{-1}$ ) LECs [45]. Therefore, at present cyanine LECs can be attractive for applications where low cost but not high performance is the prime focus. 


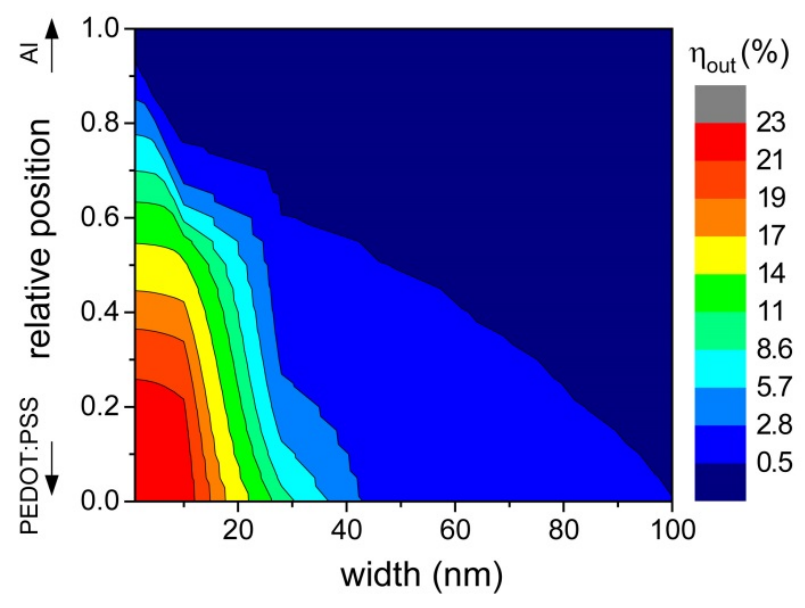

Figure 6. Simulated outcoupling efficiency for a Gaussian emission zone as a function of central position and full width half maximum.

\section{CONCLUSION}

LECs are one of the simplest kinds of electroluminescent devices and the prospect of lowcost, large-area and simple solution-processed emissive light fixtures is appealing. For that purpose, we investigated a series of cyanine host-guest systems for applications in visible emitting LECs.

Pure films of cyanines exhibited pronounced non-radiative quenching due to molecular aggregation, resulting in low PLQE. By PL studies we demonstrated resonant energy transfer between excited cyanine host and cyanine guest molecules. The energy transfer process was efficient because the exceptionally high extinction coefficient of cyanines can compensate for the low host PLQE. The PL in the best-performing host-guest blend increased by up to a factor of 15 and resulted in a PLQE of $16.2 \%$. Admittedly, this is a modest value for lighting applications and suggests that the non-radiative quenching processes were reduced among host and guest cyanines, but not completely eliminated. In other host-guest systems the PL gain and guest PLQE in the visible $(\leq 7 \%)$ were even smaller. A low PLQE increase was also observed when the energy transfer to the guest was accompanied by an efficient transfer to an 
emissive host dimer. In these situations, the calculated Förster radii overestimated the actual gain in PLQE.

We found that the LEC efficiency increase for host-guest systems directly correlated with the PLQE enhancement. For the red-emitting 3b:3d system the best experimental EQE was $0.36 \%$ and the efficiency decreased only slightly when increasing the current density. The maximum calculated EQE in this system is $0.81 \%$. The experimental and maximum EQE values are in good agreement, considering the uncertainties of the effective radiative and outcoupling efficiencies. Especially when the emission zone is too broad or close to the metal electrode, $\eta_{\text {out }}$ decreases strongly. In a LEC light emission occurs from the intrinsic (i) region that is sandwiched between n- and p-doped layers, but the developing $\mathrm{p}-\mathrm{i}-\mathrm{n}$ situation is not a priori known. Although we recently developed a method to determine the emission center in sandwich LECs [15], the width of the emission zone remains elusive because this width must not correspond to the width of the low-conductive intrinsic region. Our findings suggest that progress in this field is possible if the pronounced non-radiative quenching among cyanine molecules in a host-guest film can be suppressed. From a chemical point of view, more lowcost higher bandgap cyanine host molecules are required, eventually allowing for cyanine host-guest LECs that emit also in the short-wavelength region of the visible.

\section{SUPPORTING INFORMATION}

Synthesis of dye 1, 3a and 3b, optical constants of dye $3 b$, film stability of dye $3 a$ and $3 b$, AFM images of fresh and stored 3a dye films, Förster integral and radius calculated using solid state extinction coefficients, PL and EL spectra of $3 b$ host and 3b:3d host-guest LECs, photoluminescence of $3 \mathrm{~b}: 3 \mathrm{c}$ blends, transient LEC behaviour, normalized EQE from LECs with different active layers, dissipated power analysis. 


\section{ACKNOWLEDGEMENTS}

Financial support from the Swiss National Science Foundation (grant number 200021_144120) and the Swiss Commission for Technology and Innovation (project perolec) is acknowledged.

\section{REFERENCES}

[1] Pei, Q. B.; Yu, G.; Zhang, C.; Yang, Y.; Heeger, A. J. Polymer Light-

Emitting Electrochemical Cells. Science 1995, 269, 1086-1088.

[2] Lee, J. K.; Yoo, D. S.; Handy, E. S.; Rubner, M. F. Thin Film Light Emitting Devices from an Electroluminescent Ruthenium Complex. Appl. Phys. Lett. 1996, 69, 1686-1688.

[3] Meier, S. B.; Tordera, D.; Pertegás, A.; Roldán-Carmona, C.; Ortí, E.; Bolink, H. J. LightEmitting Electrochemical Cells: Recent Progress and Future Prospects. Mater. Today 2014, $17,217-223$.

[4] Sun, Q. J.; Li, Y. F.; Pei, Q. B. Polymer Light-Emitting Electrochemical Cells for HighEfficiency Low-Voltage Electroluminescent Devices. J. Display Technol. 2007, 3, 211-224.

[5] Slinker, J. D.; De Franco, J. A.; Jaquith, M. J.; Silveira, W. R.; Zhong, Y.-W.; MoranMirabal, J. M.; Craighead, H. G.; Abruña, H. D.; Marohn, J. A.; Malliaras, G. G. Direct Measurement of the Electric-Field Distribution in a Light-Emitting Electrochemical Cell. Nat. Mater. 2007, 6, 894-899.

[6] Pei, Q.; Heeger, A. J. Operating Mechanism of Light-Emitting Electrochemical Cells. Nat. Mater. 2008, 7, 167.

[7] van Reenen, S.; Matyba, P.; Dzwilewski, A.; Janssen, R. A. J.; Edman, L.; Kemerink, M. A Unifying Model for the Operation of Light-Emitting Electrochemical Cells. J. Am. Chem. Soc. 2010, 132, 13776-13781. 
[8] Costa, R. D.; Ortí, E.; Bolink, H. J.; Monti, F.; Accorsi, G.; Armaroli, N. Luminescent Ionic Transition-Metal Complexes for Light-Emitting Electrochemical Cells. Angew. Chem., Int. Ed. 2012, 51, 8178-8211.

[9] van Reenen, S.; Akatsuka, T.; Tordera, D.; Kemerink, M.; Bolink, H. J. Universal Transients in Polymer and Ionic Transition Metal Complex Light-Emitting Electrochemical Cells. J. Am. Chem. Soc. 2013, 135, 886-891.

[10] Shin, J. H.; Dzwilewski, A.; Iwasiewicz, A.; Xiao, S.; Fransson, A.; Ankah, G. N.; Edman, L. Light Emission at $5 \mathrm{~V}$ from a Polymer Device with a Millimeter-Sized Interelectrode Gap. Appl. Phys. Lett. 2006, 89, 013509.

[11] Carvalho, L. M.; Santos, L. F.; Guimarães, F. E. G.; Gonçalves, D.; Gomes, A. S.; Faria, R. M. Morphology of 2,5-Substituted Poly(p-phenylene vinylene) with Oligo(ethylene oxide) Side Chains/PEO-Salt Blends. Synth. Met. 2001, 119, 361-362.

[12] Tang, S.; Tan, W.-Y.; Zhu, X.-H.; Edman, L. Small-Molecule Light-Emitting Electrochemical Cells: Evidence for In Situ Electrochemical Doping and Functional Operation. Chem. Commun. 2013, 49, 4926-4928.

[13] Pertegás, A.; Tordera, D.; Serrano-Pérez, J. J.; Ortí, E.; Bolink, H. J. Light-Emitting Electrochemical Cells using Cyanine Dyes as the Active Components. J. Am. Chem. Soc. 2013, $135,18008-18011$.

[14] Wong, M. Y.; Hedley, G. J.; Xie, G. H.; Kölln, L. S.; Samuel, I. D. W.; Pertegás, A.; Bolink, H. J.; Zysman-Colman, E. Light-Emitting Electrochemical Cells and SolutionProcessed Organic Light-Emitting Diodes Using Small Molecule Organic Thermally Activated Delayed Fluorescence Emitters. Chem. Mater. 2015, 27, 6535-6542.

[15] Jenatsch, S.; Wang, L.; Bulloni, M.; Véron, A. C.; Ruhstaller, B.; Altazin, S.; Nüesch, F.; Hany, R. Doping Evolution and Junction Formation in Stacked Cyanine Dye Light-Emitting Electrochemical Cells. ACS Appl. Mater. Interfaces 2016, 8, 6554-6562. 
[16] Chen H.-F.; Liao C.-T.; Su H.-C.; Yeh Y.-S.; Wong K.-T. Highly efficient exciplex emission in solid-state light-emitting electrochemical cells based on mixed ionic holetransport triarylamine and ionic electron-transport 1,3,5-triazine derivatives. J. Mater. Chem. C 2013, 1, 4647- 4654 .

[17] Weber M. D.; Adam M.; Tykwinski R. R.; Costa R. D. Controlling the Chromaticity of Small-Molecule Light-Emitting Electrochemical Cells Based on TIPS-Pentacene. Adv. Funct. Mater. 2015, 25, 5066-5074.

[18] Subeesh M. S.; Shanmugasundaram K.; Sunesh C. D.; Won Y. S.; Choe Y. Utilization of a phenanthroimidazole based fluorophore in light-emitting electrochemical cells. J. Mater. Chem. C 2015, 3, 4683-4687.

[19] Ertl C. D.; Bolink H. J.; Housecroft C. E.; Constable E. C.; Ortí E.; Junquera-Hernández J. M.; Neuburger M.; Shavaleev N. M.; Nazeeruddin M. K.; Vonlanthen D. Bis-Sulfone- and Bis-Sulfoxide-Spirobifluorenes: Polar Acceptor Hosts with Tunable Solubilities for BluePhosphorescent Light-Emitting Devices. Eur. J. Org. Chem. 2016, 2037-2047.

[20] Hosseini, A. R.; Koh, C. Y.; Slinker, J. D.; Flores-Torres, S.; Abruña, H. D.; Malliaras, G. G. Addition of a Phosphorescent Dopant in Electroluminescent Devices from Ionic Transition Metal Complexes. Chem. Mater. 2005, 17, 6114-6116.

[21] Su, H. C.; Hsu, J. H. Improving the Carrier Balance of Light-Emitting Electrochemical Cells based on Ionic Transition Metal Complexes. Dalton Trans. 2015, 44, 8330-8345.

[22] Liao, C. T.; Chen, H. F.; Su, H. C.; Wong, K. T. Tailoring Balance of Carrier Mobilities in Solid-State Light-Emitting Electrochemical Cells by Doping a Carrier Trapper to Enhance Device Efficiencies. J. Mater. Chem. 2011, 21, 17855-17862.

[23] Su, H. C.; Wu, C. C.; Fang, F. C.; Wong, K. T. Efficient Solid-State Host-Guest LightEmitting Electrochemical Cells Based on Cationic Transition Metal Complexes. Appl. Phys. Lett. 2006, 89, 261118. 
[24] Tang, S.; Buchholz, H. A.; Edman, L. On the Selection of a Host Compound for Eefficient Host-Guest Light-Emitting Electrochemical Cells. J. Mater. Chem. C 2015, 3, 8114-8120.

[25] Chen, F. C.; Yang, Y.; Pei, Q. Phosphorescent Light-Emitting Electrochemical Cell. Appl. Phys. Lett. 2002, 81, 4278-4280.

[26] Ho, C. C.; Chen, H. F.; Ho, Y. C.; Liao, C. T.; Su, H. C.; Wong, K. T. Phosphorescent Sensitized Fluorescent Solid-State Near-Infrared Light-Emitting Electrochemical Cells. Phys. Chem. Chem. Phys. 2011, 13, 17729-17736.

[27] Pertegás, A.; Wong, M. Y.; Sessolo, M.; Zysman-Colman, E.; Bolink, H. J. Efficient Light-Emitting Electrochemical Cells Using Small Molecular Weight, Ionic, Host-Guest Systems. ECS J. Solid State Sci. Technol. 2016, 5, R3160-R3163.

[28] Anantharaman, S. B.; Yakunin, S.; Peng, C.; Vismara, M. V. G.; Graeff, C. F. O.; Nüesch, F. A.; Jenatsch, S.; Hany, R.; Kovalenko, M. V.; Heier, J. Strongly Red-Shifted Photoluminescence Band Induced by Molecular Twisting in Cyanine (Cy3) Dye Films. J. Phys. Chem. C 2017, 121, 9587-9593.

[29] Powell, R. C.; Soos, Z. G. Singlet Exciton Energy-Transfer in Organic Solids. J. Lumin. 1975, 11, 1-45.

[30] Castro, F. A.; Benmansour, H.; Moser, J.-E.; Graeff, C. F. O.; Nüesch, F.; Hany, R. Photoinduced Hole-Transfer in Semiconducting Polymer/Low-Bandgap Cyanine Dye Blends: Evidence for Unit Charge Separation Quantum Yield. Phys. Chem. Chem. Phys. 2009, 11, 8886-8894.

[31] Klàn, P.; Wirz, J. Photochemistry of Organic Compounds: From Concepts to Practice; John Wiley \& Sons Ltd: Chichester, UK, 2009.

[32] AlTal, F.; Gao, J. Long-Term Testing of Polymer Light-Emitting Electrochemical Cells: Reversible Doping and Black Spots. Org. Electr. 2015, 18, 1-7. 
[33] Tordera, D.; Frey, J.; Vonlanthen, D.; Constable, E.; Pertegás, A.; Ortí, E.; Bolink, H. J.; Baranoff, E.; Nazeeruddin, M. K. Low Current Density Driving Leads to Efficient, Bright, and Stable Green Electroluminescence. Adv. Energy Mater. 2013, 3, 1338-1343.

[34] Suhr, K. J.; Bastatas, L. D.; Shen, Y. L.; Mitchell, L. A.; Holliday, B. J.; Slinker, J. D. Enhanced Luminance of Electrochemical Cells with a Rationally Designed Ionic Iridium Complex and an Ionic Additive. ACS Appl. Mater. Interfaces 2016, 8, 8888-8892.

[35] Wu, Y.; Wu, H. R.; Zhou, Y. C.; Zhan, Y. Q.; Zhou, J.; Ding, X. M.; Hou, X. Y. Excitation Energy Transfer Between Tris-(8-hydroxyquinoline) Aluminum and a Red Dye. Appl. Phys. Lett. 2006, 88, 123512.

[36] Scully, S. R.; Armstrong, P. B.; Edder, C.; Frechet, J. M. J.; McGehee, M. D. LongRange Resonant Energy Transfer for Enhanced Exciton Harvesting for Organic Solar Cells. Adv. Mater. 2007, 19, 2961-2966.

[37] Palilis, L. C.; Melinger, J. S.; Wolak, M. A.; Kafafi, Z. H. Excitation Energy Transfer in Tris(8-hydroxyquinolinato)aluminum Doped with a Pentacene Derivative. J. Phys. Chem. B 2005, 109, 5456-5463.

[38] Stender, B.; Völker, S. F.; Lambert, C.; Pflaum, J. Optoelectronic Processes in Squaraine Dye-Doped OLEDs for Emission in the Near-Infrared. Adv. Mater. 2013, 25, 2943-2947.

[39] Jenatsch, S.; Hany, R.; Véron, A. C.; Neukom, M.; Züfle, S.; Borgschulte, A.; Ruhstaller, B.; Nüesch, F. Influence of Molybdenum Oxide Interface Solvent Sensitivity on Charge Trapping in Bilayer Cyanine Solar Cells. J. Phys. Chem. C 2014, 118, 17036-17045.

[40] Tang, S.; Edman, L. Quest for an Appropriate Electrolyte for High-Performance LightEmitting Electrochemical Cells. J. Phys. Chem. Lett. 2010, 1, 2727-2732.

[41] Tsutsui, T.; Aminaka, E.; Lin, C. P.; Kim, D. U. Extended Molecular Design Concept of Molecular Materials for Electroluminescence: Sublimed-Dye Films, Molecularly Doped Polymers and Polymers with Chromophores. Phil. Trans. R. Soc. A 1997, 355, 801-814. 
[42] Gao, J.; Yu, G.; Heeger, A. J. Polymer Light-Emitting Electrochemical Cells with Frozen p-i-n Junction. Appl. Phys. Lett. 1997, 71, 1293-1295.

[43] Greenham, N. C.; Friend, R. H.; Bradley, D. D. C. Angular Dependence of the Emission from a Conjugated Polymer Light-Emitting Diode: Implications for Efficiency Calculations. Adv. Mater. 1994, 6, 491-494.

[44] Brütting, W.; Frischeisen, J.; Schmidt, T. D.; Scholz, B. J.; Mayr, C. Device Efficiency of Organic Light-Emitting Diodes: Progress by Improved Light Outcoupling. Phys. Status Solidi A 2013, 210, 44-65.

[45] Mindemark J.; Tang S.; Wang J.; Kaihovirta N.; Brandell D.; Edman L. HighPerformance Light-Emitting Electrochemical Cells by Electrolyte Design. Chem. Mater. 2016, 28, 2618-2623. 